\title{
MHD turbulence in a channel with spanwise field
}

\author{
Dmitry Krasnov * 1 , Oleg Zikanov ${ }^{2}$, Joerg Schumacher ${ }^{1}$, and Thomas Boeck ${ }^{1}$ \\ ${ }^{1}$ Department of Mechanical Engineering, Ilmenau University of Technology, P.O. Box 100565, 98684 Ilmenau, Germany \\ 2 Department of Mechanical Engineering, University of Michigan - Dearborn, Dearborn MI 48128-1491, USA
}

\begin{abstract}
We study turbulent channel flow of an electrically conducting liquid with a homogeneous magnetic field imposed in the spanwise direction. The Lorentz force is modelled using the quasistatic approximation. Direct and large-eddy simulations are performed for hydrodynamic Reynolds numbers $\mathrm{Re}=10000$ and $\mathrm{Re}=20000$ and the Hartmann number varying in a wide range. The main effect of the magnetic field is the suppression of turbulent velocity fluctuations and momentum transfer in the wall-normal direction. Comparing the results from direct and large-edddy simulations we show that the dynamic Smagorinsky model accurately reproduces the flow transformation.
\end{abstract}

(c) 2008 WILEY-VCH Verlag GmbH \& Co. KGaA, Weinheim

\section{Introduction}

A channel flow in a purely spanwise magnetic field can be viewed as a generalized model of flows in the presence of a magnetic field with non-zero component parallel to solid walls. Such flows can be found in numerous metallurgical and materials processing applications, e.g. the electromagnetic flow control in continuous casting [1,2] and growth of large silicon crystals. Another area of applications is the liquid metal cooling blanket for fusion reactors [3]. Instability and transition to turbulence in sidewall boundary layers (in respect to which the magnetic field is spanwise) is one of the possible ways to achieve the desired intensification of heat and mass transfer. Lee \& Choi [4] have shown that a spanwise magnetic field can lead to substantial turbulent drag reduction in a channel flow. Recently, we have studied transition to turbulence in this configuration at subcritical Reynolds numbers [5]. Here we extend these numerical studies to turbulence at supercritical Reynolds numbers. On the one hand, we examine the flow transformation caused by the spanwise magnetic field. The changes of turbulence statistics, wall friction, and coherent structures are documented. On the other hand, we examine the performance of the classical and dynamic Smagorinsky models for the large-eddy simulation (LES) of channel flow with spanwise magnetic field by comparison with direct numerical simulations (DNS).

\section{Computational model}

We consider the flow of an incompressible electrically conducting fluid in an infinite plane channel between insulating walls located at $z= \pm D$ with a magnetic field $B_{0}$ in the spanwise direction. The flow is driven by a pressure gradient in the streamwise direction such that the volume flux $Q$ remains constant. We apply the quasi-static approximation, whereby the fluctuations of the magnetic field arising due to the fluid motion adjust instantaneously to the velocity fluctuations and are much weaker than the imposed magnetic field. The governing equations reduce to the Navier-Stokes system with the additional Lorentz force. The length and velocity scales are the laminar centerline velocity $U$ and the channel half width $D$. The unit of time is $D / U$. Nondimensional parameters are the Reynolds number $R e=U D / \nu$ and the Hartmann number

$$
H a=B_{0} D \sqrt{\frac{\sigma}{\rho \nu}} .
$$

For the numerical simulations we use a pseudo-spectral Fourier-Chebshev method, where the flow field is represented by velocity potentials complying with the incompressibility constraint. A description of the algorithm and the corresponding flow solver is given elsewhere [5]. The algorithm is parallelized using domain decomposition in the streamwise direction. Fourier transforms are performed locally by transposition of the data array across the processors.

\section{Results}

Table 1 lists the parameters of the DNS and LES runs. The Hartmann number was increased until the turbulence could no longer be sustained. For the two values of $R e$, the mean friction coefficient $c_{f}$ at the largest $H a$ decreased by about $30 \%$ (relative to its value at $H a=0$ ). At the same time, the mean velocity profile becomes steeper upon increasing $H a$, as seen in Fig. 1 for $R e=10^{4}$. One can also see that the logarithmic region is lost at $H a>0$ as a result of the magnetic damping of the turbulence. Correspondingly, the Reynolds stresses decrease with increasing $\mathrm{Ha}$. The same observations apply for

\footnotetext{
* Corresponding author: e-mail: dmitry.krasnov@tu-ilmenau.de, Phone: +493677692429, Fax: +49367769 1281
} 


\begin{tabular}{cccccc}
$R e$ & $R e_{Q}$ & $\mathrm{Ha}$ & DNS: $N_{x} \times N_{y} \times N_{z}$ & LES: $N_{x} \times N_{y} \times N_{z}$ & $L_{x} \times L_{y} \times L_{z}$ \\
\hline 10000 & $6.67 \times 10^{3}$ & $0,10,20,30$ & $256 \times 256 \times 256$ & $64 \times 64 \times 64$ & $2 \pi \times \pi \times 2$ \\
20000 & $1.33 \times 10^{4}$ & $0,20,30,40$ & $512 \times 512 \times 256$ & $128 \times 128 \times 128,64 \times 64 \times 64$ & $2 \pi \times \pi \times 2$
\end{tabular}

Table 1 Parameters of DNS and LES. The parameters $L_{i}$ denote the dimensions of the computational domain and $N_{i}$ the corresponding numbers of collocation points. $R e_{Q}$ is the flux-based Reynolds number.

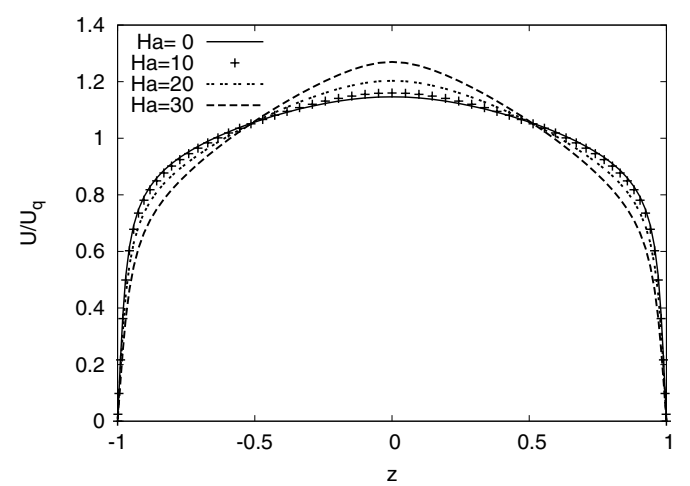

a)

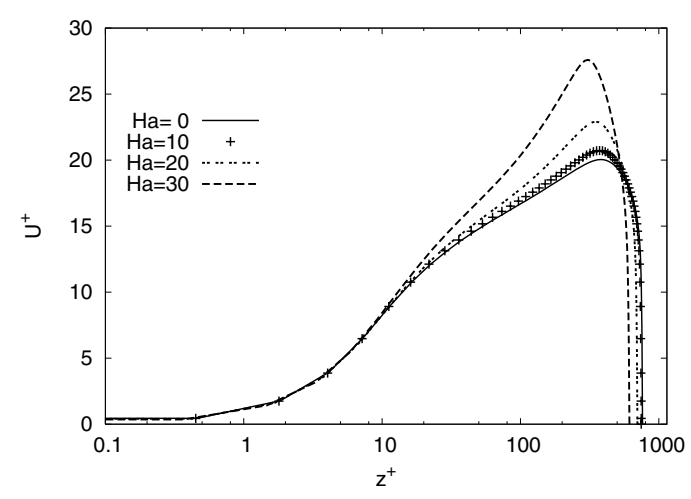

b)

Fig. 1 Effect of the magnetic field on the flow properties. Results of DNS are shown for $R e=10^{4}$. (a) Mean velocity profiles in global units. (b) Mean velocity profiles in friction units.
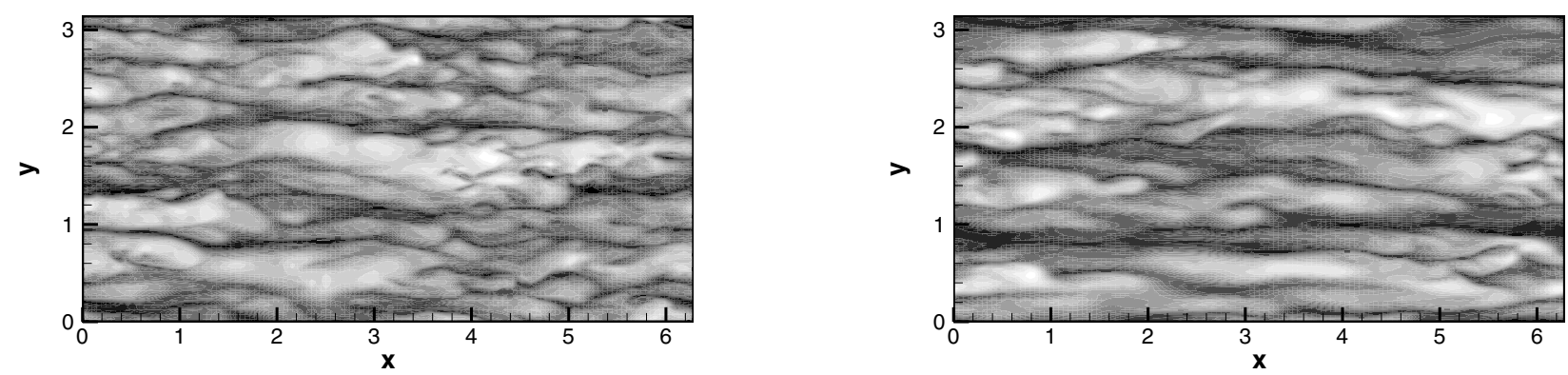

a)

Fig. 2 Effect of magnetic field on coherent structures of the flow at $R e=10^{4}$ : Contour plots of the streamwise velocity fluctuations (from DNS) at $H a=0(a)$ and at $H a=30(b)$.

$R e=2 \times 10^{4}$. Flow structures in the near-wall region are visualized in Fig. 2 by contours of streamwise velocity perturbations at $z=0.95 D$. The fields are normalized by their rms values averaged over the horizontal plane. Upon increasing $H a$, the near-wall streaks become more stable (less susceptible to small-scale perturbations) and wider in the spanwise direction. These observations are also in agreement with the earlier results by Lee and Choi [4]. For one-point correlations, the comparison of LES with DNS shows generally good agreement for the dynamic Smagorinsky model, whereas the classical Smagorinsky model deviates from DNS for larger values of $\mathrm{Ha}$. Velocity gradient statistics are less well reproduced by the LES.

Acknowledgements TB, DK and OZ acknowledge financial support from the Deutsche Forschungsgemeinschaft (Emmy-Noether grant Bo 1668/2-3 and Gerhard-Mercator visiting professorship program). OZ acknowledges support from the U.S. Department of Energy (grant DEFG02 03 ER46062). Computer resources were provided by the computing center of TU-Ilmenau and of the Forschungszentrum Jülich (NIC).

\section{References}

[1] Davidson P.A.: Magnetohydrodynamics in materials processing. Annu. Rev. Fluid Mech. 31:273-300, 1999.

[2] Thomas B.G., Zhang L.: Mathematical modeling of fluid flow in continuous casting: a Review. ISIJ Intern. 41:1181, 2001.

[3] Barleon L., Burr U., Mack K.J., Stieglitz R. Fusion Techn. 39(2):127, 2001.

[4] Lee D., Choi, H.: Magnetohydrodynamic turbulent flow in a channel at low magnetic Reynolds number, J. Fluid Mech. 439: 367-394, 2001.

[5] Krasnov D., Rossi M., Zikanov O., Boeck T.: Optimal growth and transition to turbulence in channel flow with spanwise magnetic field. J. Fluid Mech. 596, 73-101, 2008. 